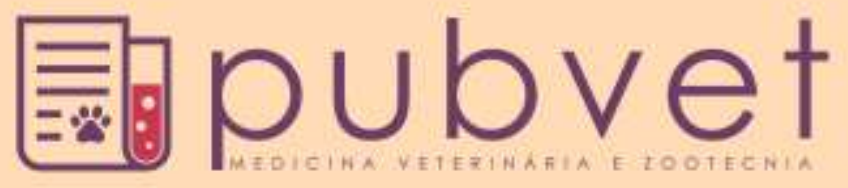

HTTP://DX.DOI.ORG/10.22256/PUBVET.V11N3.267-271

\title{
Eimeriose em bovinos leiteiros do município de Silveiras - SP
}

\author{
Matheus Diniz Gonçalves Coêlho ${ }^{1 *}$, Jessika Calheiros Ribeiroº ${ }^{2}$ Kaio Sendretti de \\ Almeida $^{2}$, Thaina Ferreira Franco' ${ }^{2}$, Lucas Tobias Rodrigues Maciel ${ }^{3}$, Fernanda Bueno \\ Sant'Anna Pereira ${ }^{3}$, Lilian Saito Ormachea Bozo $^{3}$, Francine Alves da Silva Coêlho ${ }^{4}$ \\ ${ }^{1}$ Docente e pesquisador do Laboratório de Parasitologia e Malacologia, Fundação Universitária Vida Cristã - FUNVIC, Pindamonhangaba, \\ São Paulo, Brasil.profmatheuscoelho@gmail.com \\ ${ }^{2}$ Discente do Curso de Farmácia, Fundação Universitária Vida Cristã - FUNVIC, Pindamonhangaba, São Paulo, Brasil. \\ jesscalheiros@yahoo.com,kaiokaic@hotmail.com, thainaferreiraf@hotmail.com \\ ${ }^{3}$ Discente do Curso de Farmácia e Estagiário do Laboratório de Parasitologia e Malacologia (LAPAM), Fundação Universitária Vida Cristã \\ - FUNVIC, Pindamonhangaba, São Paulo,Brasil.ltrmaciel@gmail.com,fernandabsp@gmail.com, liliaan.saito@hotmail.com, \\ ${ }^{4}$ Professora e Pesquisadora do Laboratório de Parasitologia, Departamento de Biociências, Universidade de Taubaté - UNITAU, Taubaté, \\ São Paulo, Brasil.francine.ascoelho@gmail.com \\ *autor para correspondência
}

RESUMO. O objetivo do presente trabalho foi efetuar um levantamento epidemiológico para determinar a ocorrência de Eimeria spp. em 82 bovinos leiteiros de uma Fazenda de médio porte no Vale do Paraíba, incluindo bezerros e bovinos adultos. Eimeria é um coccídeo que ataca principalmente o epitélio digestivo de aves e mamíferos domésticos, sendo um importante agente causador de diarreias prejudiciais a estes animais. Essa doença acaba acometendo os bezerros, e, por serem mais sensíveis à infecção, eles apresentam sintomas como diarreia sanguinolenta, fraqueza, perda de peso, entre outros. Sua transmissão pode ocorrer pela ingestão de oocistos, que são encontrados em água contaminada, pastagens ou até mesmo na pelagem dos animais. O diagnóstico é feito através dos sinais clínicos, lesões macroscópicas e por meio da realização de exames de fezes, evidenciando oocistos do parasito. Após análise de amostras fecais coletadas diretamente da ampola retal dos animais, observou-se uma ocorrência elevada de Eimeria spp. tanto nos bovinos adultos (50\%), quanto nos bezerros (62\%), sendo maior $(\mathrm{P}<0,05)$ em relação a outros protozoários e até mesmo em relação aos helmintos, podendo levar a perdas econômicas, decorrentes da morte de bezerros e perda de produção, trazendo a tona não só a necessidade de tratamento e manejo adequado do rebanho estudado, como também a necessidade da realização de inquéritos semelhantes, visando minimizar os impactos negativos decorrentes dessa importante parasitose bovina.

Palavras chave: Bovino, Eimeria spp

\section{Eimeriosis in dairy cattle of the municipality of Silveiras - SP}

ABSTRACT. The purpose of this study was to present the results of an epidemiological survey to determine possible incidents of Eimeria spp in 82 dairy cattle, including calves and adult cattle, living in a medium-sized farm in Vale do Paraíba. Known as coccidian; which normally attacks the digestive epithelium of birds and domestic mammals, Eimeria is the major responsible agent that causes diarrhea harmful to these animals. Being more susceptible to infections, the calves are most affected by the disease; they show symptoms such as bloody diarrhea, weakness, weight loss, and others. The transmission can occur through ingestion of oocysts which are found in contaminated water, pastures or even the coat of the animal. Clinical signs such as gross lesions and also examinations on feces can determine if there is any sign of the parasite oocysts. After collecting fecal samples directly from the rectum of those animals, it was observed that there was a high incidence of Eimeria spp both in adult cattle (50\%), calves (61.5\%), and it was significantly higher ( $\mathrm{p}<$ 0.05 ) compared to other protozoa and even to helminthes, leading to economic losses, 
arising from the death of calves and loss of production, which highlights not only the need for treatment and proper management of the studied herd, as well as the need to carry out similar surveys in order to minimize the negative impacts of this important bovine parasitizes.

Key words: Cattle herd, Eimeria spp

\section{Eimeriosis en bovinos lecheros en la ciudad de Silveiras - SP}

El objetivo de este estudio fue realizar un estudio epidemiológico para determinar la ocurrencia de Eimeria spp. en 82 vacas lecheras de una granja de tamaño medio en el Valle del Paraíba, incluyendo los machos adultos y terneros. Eimeria es un coccidio que ataca principalmente el epitelio digestivo de aves y mamíferos domésticos, siendo un importante agente causal de la diarrea perjudicial para estos animales. Esta enfermedad afecta a los terneros y por ser más susceptibles a la infección, muestran signos tales como diarrea sanguinolenta, debilidad, pérdida de peso, entre otros. Su transmisión puede ocurrir a través de la ingestión de ooquistes que se encuentran en aguas contaminadas, pastos o inclusos en el pelaje del animal. El diagnóstico se realiza por signos clínicos, lesiones macroscópicas y por los exámenes de heces, que muestran los ooquistes del parásito. Tras el análisis de muestras fecales recogidas directamente del recto de los animales, hubo una alta prevalencia de Eimeria spp. tanto en los bovinos adultos (50\%), como en los terneros $(61.5 \%)$ que fue significativamente mayor $(\mathrm{p}<0,05)$ en comparación con otros protozoos e incluso en relación con los helmintos, pudiendo conducir a pérdidas económicas, resultantes de la muerte de terneros y la pérdida de producción, trayendo a tono no solamente la necesidad de tratamiento y manejo adecuado del hato estudiado, así como la necesidad de llevar a cabo estudios similares, con el fin de minimizar los impactos negativos de este importante parasitosis bovina.

Palabras clave: Bovino, Eimeria spp

\section{Introdução}

Eimeria é um parasito do epitélio digestivo de artrópodes e vertebrados, sendo o principal causador da diarreia em bovinos. A diarreia é uma das principais doenças que acomete os bezerros, ocasionando perdas econômicas (Lima, 2004).

Alguns fatores são importantes para ocorrência de eimeriose, dentre os quais: a temperatura, deficiência nutricional, reagrupamento dos animais, desmame precoce e doenças infecciosas. Os animais adultos assintomáticos são fontes de infecção para os bezerros recém-nascidos, que podem contrair a infecção poucos dias após o nascimento (Blanco, 2015).

Os bezerros são os mais sensíveis à infecção devido a diversos fatores predisponentes relacionados com má ingestão do colostro, dificuldades de adaptação ao ambiente e da falta de uma resposta imune específica para proteção contra a doença (Polizel, 2013).

A intensidade de infecção é maior na estação chuvosa, pois os a alimentação na pastagem eleva as chances de ingestão de oocistos em relação as calhas de alimentação. O estresse ambiental, social e fisiológico como desagrupamento, desnutrição e desmame, podem interferir na eficiência da resposta imune, podendo ser responsáveis pela ocorrência de surtos de coccidiose (Amato Neto et al., 1996).

A transmissão se dá pela ingestão de oocistos esporulados os quais podem ser ingeridos por bezerros quando se agrupam em torno de comedouros e bebedouros, podendo inclusive se contaminar ao lamberem a pelagem e forragem sujas de fezes (Nelson and Couto, 2015, Vidal et al., 2014).

A idade da primeira infecção dos bezerros por Eimeria spp. varia de acordo com as condições sanitárias da propriedade, porém, observa-se que tal parasitose ocorre nas primeiras semanas de vida e a eliminação de oocistos nas fezes em animais a partir da terceira semana (Danta (Dantas et al., 2015).

No presente trabalho objetivou-se efetuar um levantamento epidemiológico para determinar a ocorrência de Eimeria spp., bem como de outras parasitoses, em bovinos leiteiros de uma fazenda no município de Silveiras, Região do Vale do Paraíba, SP. 


\section{Material e Métodos}

As coletas das amostras fecais foram realizadas no período de fevereiro de 2015 a março de 2015, sendo coletadas 26 amostras de bezerros de ambos os sexos, de 0 a 9 meses de idade e 56 amostras de bovinos adultos de 24 meses a 96 meses de idade, ambos sendo da raça Girolando e de criação de uma única fazenda, sendo comumente alimentados no pasto. Com auxilio de espátulas e luvas descartáveis, as amostras foram transferidas para coletores universais estéreis previamente identificados com nome ou número de registro de cada animal, onde foram armazenadas, e transportadas sob refrigeração em caixas de isopor, para serem processadas no laboratório de parasitologia da FUNVIC - Faculdade de Pindamonhangaba.

Todas as amostras fecais foram coletadas da fazenda Leite no Asfalto (22 $399^{\prime} 52.8^{\prime \prime} \mathrm{S}$ $\left.44^{\circ} 58^{\prime} 20.8^{\prime \prime} \mathrm{W}\right)$, localizada na cidade de Silveiras no interior do estado de São Paulo.

Para realização dos exames coproparasitológicos foi empregado o método de Ritchie modificado (1948), que se fundamenta na centrifugo-sedimentação de formas parasitárias em solução de formalina-acetato de etila (Almeida et al., 2009). Também foi realizada a coloração de Kinyoun, objetivando pesquisa de Cryptosporidium, outro parasito que pode causar diarreia em bovinos (Amato Neto et al., 1996). Em acréscimo, as amostras foram processadas pelo método de Sheather, que consiste no centrífugoflutuação de formas evolutivas de parasitos em solução de sacarose (densidade $=1,2$ ).

Com o intuito de observar diferenças significativas na ocorrência dos parasitos diagnosticados, os resultados foram avaliados estatisticamente, utilizando o teste de KruskalWallis, seguido do teste de Student-NewmanKeuls, ao nível de significância de 5\%.

\section{Resultados}

Após realização dos exames coproparasitológicos, observou-se uma elevada ocorrência do protozoário comensal $E$. nana $(60,7 \%)$ e de Eimeria spp. (50\%) no rebanho, não havendo diferença na frequência com relação às duas faixas etárias avaliadas, conforme observado nas tabelas 1 e 2 .

Também se observou uma elevada ocorrência de Strongyloides spp. no rebanho avaliado
(Tabelas 1 e 2); porém, com predominância maior $(p=0,0009)$ em bezerros.

Tabela 1. Prevalência de parasitos intestinais e comensais em bovinos adultos $(n=56)$ em rebanho do Município de Silveiras, SP (2015).

\begin{tabular}{lccc}
\hline Parasitos & Positivas & $\begin{array}{c}\text { Ritchie } \\
\text { modificado }\end{array}$ & Sheathers's \\
\hline Endolimax nana & $34(60,7 \%)$ & $34(60,7 \%)$ & $6(10,7 \%)$ \\
Eimeria spp. & $28(50,0 \%)$ & $26(46,4 \%)$ & $20(35,7 \%)$ \\
Strongyloides spp. & $2(3,7 \%)$ & $1(1,8 \%)$ & $1(1,8 \%)$ \\
Entamoeba coli & $19(33,9 \%)$ & $10(17,8 \%)$ & $13(23,2 \%)$ \\
\hline
\end{tabular}

Tabela 2. Prevalência de parasitos intestinais e comensais em bezerros $(n=26)$ em rebanho do Município de Silveiras, SP. (2015)

\begin{tabular}{lccc}
\hline Parasitos & Positivas & $\begin{array}{c}\text { Ritchie } \\
\text { modificado }\end{array}$ & Sheathers's \\
\hline Eimeria spp. & $16(61,5 \%)$ & $16(61,5 \%)$ & $16(61,5 \%)$ \\
Strongyloides spp. & $11(42,3 \%)$ & $3(11,5 \%)$ & $9(34,6 \%)$ \\
Trichuris spp. & $6(23,1 \%)$ & $4(15,4 \%)$ & $6(23,1 \%)$ \\
\hline
\end{tabular}

Já com relação aos métodos utilizados para realização dos exames coproparasitológicos, a saber, os métodos de Ritchie e Sheather, observou-se que o método de Sheather foi superior ao método de Ritchie, para pesquisa de ovos de Strongyloides spp. $(\mathrm{p}=0,0233)$, por outro lado, o método de Ritchie se mostrou superior ( $\mathrm{p}<$ $0,0001)$ ao método de Sheather para pesquisa de Endolimax nana, conforme observado na tabela 3.

Tabela 3. Comparação entre dois métodos de exame coproparasitológico para o diagnóstico de enteroparasitoses de bovinos, 2015.

\begin{tabular}{lcc}
\hline Parasitos & Ritchie modificado & Sheather's \\
\hline Endolimax nana & $34(41,5 \%)^{*}$ & $06(7,3 \%)$ \\
Eimeria spp. & $32(39,0 \%)$ & $36(43,9 \%)$ \\
Strongyloides spp. & $04(4,9 \%)$ & $10(12,2 \%)^{*}$ \\
Entamoeba coli & $10(12,2 \%)$ & $13(15,8 \%)$ \\
Trichuris spp. & $04(4,9 \%)$ & $06(7,3 \%)$ \\
\hline
\end{tabular}

\section{Discussão}

A elevada ocorrência de Eimeria spp. observado no presente trabalho correspondem com outras pesquisas feitas com bovinos leiteiros, dentre os quais, os resultados apresentados por Dantas et al. (2015) que demonstraram uma elevada ocorrência de Eimeria spp., a saber, $46,7 \%$ de amostras fecais positivas em bezerros.

A Eimeriose tem uma grande relevância para o rebanho bovino, sendo que suas maiores implicações são decorrentes da infecção em bezerros, uma vez que está intimamente relacionada com a imunidade do hospedeiro. Os 
bezerros se encontram em uma faixa etária susceptível, sendo considerados frágeis em termos imunológicos, já que comumente ingerem pouco colostro, e são submetidos a alto nível de estresse e risco de subnutrição, fatores estes que podem contribuir para uma maior predisposição a parasitose (Polizel, 2013).

Outro fator que influencia na ocorrência de Eimeriose está relacionada a falta de higiene dos locais onde os animais comem e bebem, favorecendo muito a contaminação por oocistos de Eimeria. Neste sentido, tanto as vacas como os bezerros podem facilmente se contaminar através da alimentação, podendo também haver uma grande probabilidade de que os bezerros possam se contaminar através do contato com os animais adultos, já que os mesmos têm o costume de lamberem a pelagem destes (Nelson and Couto, 2015, Vidal et al., 2014).

É provável que a elevada ocorrência de Strongyloides spp. também possa estar relacionada com a baixa imunidade e com a ingestão insuficiente de colostro, o que pode influenciar nos mecanismos de proteção imune frente a este helminto.

A elevada ocorrência de Strongyloides spp. nos bezerros (Tabela 2) vai de encontro a evidências da literatura científica, segundo as quais, a maioria dos pesquisadores apontam como sendo o parasito mais frequente entre bezerros, podendo também ser responsável por retardo no crescimento, baixas na produção leiteira e morte em bezerros recém nascidos (Silva et al., 2001).

A ausência de amostras positivas para Cryptosporidium spp. corrobora com os apresentados por outros pesquisadores, dentre os quais (Cardoso et al., 2008). Tais autores realizaram um inquérito coproparasitológico para determinar a ocorrência de Cryptosporidium spp. em um rebanho bovino leiteiro no Município de Caçapava, também situado no Vale do Paraíba e observaram uma baixa frequência de amostras positivas, que variou de 0 a $1,4 \%$.

Embora este parasito seja de importância para a Saúde veterinária há uma relevância para a Saúde pública, uma vez que pode levar a diarreia grave, particularmente em indivíduos imuno comprometidos (Nunes, 2014), o fato de se tratar de um protozoário oportunista pode justificar a sua baixa ocorrência, que, conforme supracitado, tem sido observada em diversos inquéritos epidemiológicos.
A maior eficácia do método de Sheather para pesquisa de Strongyloides spp. já era esperada, uma vez que este método é mais indicado para pesquisa de formas evolutivas de baixa densidade. Por outro lado, a solução saturada de sacarose pode ter influenciado de forma negativa para pesquisa de Endolimax nana, devido à possível perda de água por osmose por parte dos cistos do protozoário, porém sendo necessário o delineamento de experimentos para confirmar tal hipótese.

\section{Conclusão}

Conclui-se que é elevada a ocorrência de Eimeria spp. e Strongyloides spp. no rebanho bovino avaliado no presente trabalho, permitindo propor ao criador a adoção de estratégias de manejo adequado do mesmo, por meio da higienização adequada dos comedouros e da forragem, para minimizar os riscos de morbidade e mortalidade por estes importantes parasitos.

Por fim, cabe inferir a necessidade da realização de futuros estudos que visem avaliar a eficácia da adoção destas medidas preventivas, como forma de auxiliar no incremento do manejo e do processo de criação deste rebanho.

\section{Referências Bibliográficas}

Almeida, A. F., Onani, K. A. A., Segura-Munoz, S. I., Santos, V. M. d. \& Takayanagui, A. M. M. 2009. Adaptação ao método de Ritchie para diagnóstico de helmintos e protozoários em amostras de lodo de esgoto com minimização de produtos químicos. Mundo Saúde, 33, 427432.

Amato Neto, V., Braz, L. M. A., Pietro, A. O. D. \& Módolo, J. R. 1996. Pesquisa de oocistos de Cryptosporidium sp em fezes: comparação entre os métodos de Kinyoun modificado e de Heine. Revista da Sociedade Brasileira de Medicina Tropical, 29, 575-578.

Blanco, Y. A. C. 2015. Efeito e custos do tratamento estratégico seletivo no controle de parasitoses gastrointestinais em bezerras leiteiras. Departamento de Zootecnia. Universidade Federal de Lavras, Minas Gerais.

Cardoso, J. M. S., Silveira, F. L., Araújo, A. J. U. S., Carvalho, J. C. C. \& Kanamura, H. Y. 2008. Ocorrência de Cryptosporidium spp. em um rebanho bovino leiteiro no município de Caçapava, estado de São Paulo, Brasil. Revista Brasileira de Parasitologia Veterinária, 17, 239-242. 
Dantas, P. C. S., Santos, L. D., Jesus, O. F., Calasans, T. A. S., Porto, A. G., Carvalho, C. D., Jeraldo, V. d. L. S., Allegretti, S. M. \& Melo, C. M. 2015. Ocorrência de parasitoses gastrintestinais em vacas leiteiras e respectivos bezerros durante o período de amamentação, na Fazenda São Paulinho, Município de Itapicuru/BA. Scientia Plena, 11.

Lima, J. D. 2004. Coccidiose dos ruminantes domésticos. Revista Brasileira de Parasitologia Veterinária, 13, 9-13.

Nelson, R. W. \& Couto, C. G. 2015. Medicina interna de pequenos animais. Elsivier Editora, Amsterdan.

Nunes, M. R. F. 2014. Rastreio de formas parasitárias em fezes de cães recolhidas em espaços públicos na cidade de Beja. Faculdade de Medicina Veterinária. Universidade de Lisboa. Faculdade de Medicina Veterinária, Lisboa.

Polizel, F. F. 2013. Controle de eimeriose em bovinos. Universidade Estadual Paulista Júlio de Mesquita Filho, Araçatuba.
Silva, L. A. F., Fioravanti, M. C. S., Dias Filho, F. C. \& Eurides, D. 2001. Sanidade dos bezerros leiteiros da concepção ao desmame. Goiânia: Talento, 1, 1-87.

Vidal, L. G. P., Fagundes, T. F. \& Pantoja, C. S. 2014. Morfometria de oocistos de" Eimeria" em bezerras segundo a faixa etária e a intensidade de infecção, Município de Piraí, RJ. Revista Brasileira de Saúde e Produção Animal, 14, 765-777.

\section{Article History:}

Received 5 December 2016

Accepted 19 January 2017

Available on line 23 January 2017

License information: This is an open-access article distributed under the terms of the Creative Commons Attribution License 4.0, which permits unrestricted use, distribution, and reproduction in any medium, provided the original work is properly cited. 